\title{
Phacoemulsification Combined with Intravitreal Triamcinolone Acetonide Injection in Cataract Patients with Diabetic Macular Edema
}

K.G.Mohamed, T.T.Soliman, A.E.Mohamed and W.S.Abusaif

Ophthalmology Dept., Faculty of Medicine, Benha Univ., Benha, Egypt

E-Mail:ahmed2356@gmail.com

\begin{abstract}
To assess the safety and efficiency of combined phacoemulsification surgery and intravitreal triamcinolone (IVTA) injection in patients with cataract and diabetic macular edema. This investigation included "40 patients" having youthful decrepit waterfall with clinically critical macular edema. Phacoemulsification was finished with back chamber intraocular focal point implantation and intravitreal triamcinolone infusion. Patients were followed up on day 1,day 3 then week after week for multi month, and from that point month to month for a half year postoperatively. Best adjusted visual keenness (BCVA) and focal macular thickness (CMT), and intraocular pressure (IOP) were recorded. All patients finished a half year of development. Mean BCVA demonstrated factually noteworthy improvement from a standard of $0.08 \pm 0.05$ decimal to $0.3 \pm 0.13$ decimal at 3 months and $0.35 \pm 0.16$ decimal at a half year. Mean CMT diminished essentially from a gauge of $504.85 \pm 109.05$ $\mu \mathrm{m}$ to $341.10 \pm 75.35 \mu \mathrm{m}$ at 3 months and $322.05 \pm 87.40 \mu \mathrm{m}$ at a half year. IOP was raised in 6 patients $(15 \%)<21$ $\mathrm{mmHg}$ one month after infusion in any case, all managed with against glaucoma drugs. No infusion or medical procedure related difficulties were experienced. Phacoemulsification with simultaneous $4 \mathrm{mg}$ IVTA infusion seems, by all accounts, to be a protected and compelling choice for overseeing diabetics with waterfall and CSMO.
\end{abstract}

Keywords: Phacoemulsification, Intravitreal triamcinolone, Cataract, Diabetic macular edema.

\section{Introduction}

Macular edema is the most successive reason for visual misfortune among patients with diabetic retinopathy [1]. It is brought about by breakdown of the internal blood retinal boundary and increment vascular porousness because of arrival of fiery middle people, for example, Prostaglandins and the Vascular Endothelium Growth Factors (VEGF) [2].

Fluorescein angiography and Optical Coherence Tomography (OCT) are useful in setting up or affirming the determination of macular oedema notwithstanding assessing the level of vascular spillage and the macular thickness [3].

Waterfall medical procedure is a fiery affront to the eye, and the danger of macular thickening after straightforward phacoemulsification may increment within the sight of visual or fundamental maladies, for example, uveitis or diabetes [4].The seriousness of retinopathy may have an impact on the visual results after simple phacoemulsification in patients with diabetes. Shaky DR with clinically critical macular edema at the hour of phacoemulsification medical procedure will in general intensify postoperative macular edema [5].

Corticosteroids are known for capacity to restrain the arachidonic pathway [6]. Triamcinolone acetonide is a corticosteroid that has been utilized as intravitreal infusions for the treatment of recalcitrant diabetic macular edema. Intravitreal infusion of Triamcinolone acetonide is productive in diminishing macular edema with a transient impact enduring as long as a half year [7]. Consolidating triamcinolone acetonide infusion with waterfall medical procedure helps in controlling diabetic macular edema in one prodecure [8].

\section{The aim of the study}

Is to evaluate the safety and efficacy of phacoemulsification combined with intravitreal Triamcinolone acetonide injection in cataract patients with diabetic macular edema.

\section{Subjects \&methods}

This was an imminent interventional study that included 40 eyes of 32 patients assessed at the outpatient facility of the Ophthalmology Department, Benha University Hospital, and determined to have juvenile feeble waterfall and non-proliferative diabetic retinopathy with macular edema. Patients were exposed to phacoemulsification with back chamber intraocular focal point implantation and intravitreal triamcinolone infusion toward the finish of medical procedure, at that point followed-up for a half year after medical procedure.

All assessments were performed by a convention that followed the precepts of the Declaration of Helsinki and that was endorsed by the Institutional Review Board and Ethics Committee of Benha University. All patients got an intensive clarification of the investigation plan and points, and were given composed educated assent.

Prohibition standards included past visual injury and visual medical procedure, past laser photocoagulation or intravitreal infusions, any critical media darkness other than waterfall, proliferative diabetic retinopathy, glaucomatous patients, macular edema brought about by tractional component or any causes other than diabetic retinopathy and cases with confused phacoemulsification with vitreous herniation because of back container crack or zonular dialysis. 


\section{Pre-operative evaluation}

All patients were subjected to the following:

-History taking (age, gender, occupation, pastmedical history, past-surgical history \& ocular history)

-Best-corrected visual acuity was assessed

-Slit lamp examination to assess the density of cataract.

-Indirect ophthalmoscopy: Fundus examination was done.

-Intraocular pressure (IOP) measurement.

- Investigations

- Laboratory: -complete blood count (CBC), fasting blood sugar, HbA1c

- Fundus Fluorescein angiography (FFA) to detect signs of vascular leakage as well as ischemia in patients who demonstrate clinically visible maculopathy.

- Optical Coherence Tomography (OCT) to document macular thickness preoperatively: In all patients, 3D macula scan protocol $512 \times 128$ (128 horizontal scan lines comprised of 512 Ascans) over a $6-\mathrm{mm}^{2}$ area centered on the fovea was conducted using Topcon 3D OCT-2000 FA, JAPAN "version 8.30".

\section{Operative procedure}

Uncomplicated Phacoemulsification and intracapsular intraocular lens implantation was performed (single-piece foldable hydrophilic acrylic IOL). At the end of the cataract surgery, $4 \mathrm{mg}$ of triamcinolone acetonide (Epirelefan $40 \mathrm{mg} / 1 \mathrm{ml}$ vial ,Eipico,EGYPT) in $0.1 \mathrm{ml}$ injected at a site $3.5 \mathrm{~mm}$ posterior to the inferotemporal limbus.

\section{Postoperative evaluation}

-Patients followed up on day 1 , day 3, then weekly for 1 month, and thereafter monthly for 6 months postoperatively.

- (BCVA) and IOP were documented in each visit.

-Macular edema was monitored by OCT and Fluorescein angiography at 3months and 6 months.

\section{Results}

The study included 40 eyes of 32 diabetic patients $[18$ males $(56.25 \%)$ and 14 females
(43.75\%)] whose age ranged from 42 to 71 years (mean $=57$ years \pm 7$)$. The right eye was affected in 19 eyes $(47.5 \%)$ while the left was affected in 21 eyes (52.5\%). The mean FBS of the whole group was $286.0 \pm 7.23 \mathrm{mg} / \mathrm{dl}$ while the mean HbA1c was 7.65 \pm 0.98 , as tabulated in Table (1). IOP was elevated in 6 patients above $21 \mathrm{mmHg}$ one month after injection, but all brought under control with anti-glaucoma medications. The mean preoperaitive IOP was 13.70 \pm 1.82 (mmHg). One month after injection, the mean IOP significantly elevated to $16.40 \pm 4.53(\mathrm{mmHg})$, however, at the $3^{\text {rd }}$ month, it was significantly reduced to $14.30 \pm 1.93(\mathrm{mmHg})$ to be nonsignificant from the preoperative level. The level of IOP continued to be stable at $14.30 \pm 1.87(\mathrm{mmHg})$ till the $6^{\text {th }}$ postoperative month, as tabulated in Table (2). and illustrated in Fig (1).

The obtained results showed a significant decrease in the central macular thickness (CMT) postoperatively up to the $3^{\text {rd }}$ month in all eyes of the study except in two eyes where the reduction of CMT wasn't that significant. At the $6^{\text {th }}$ month postoperatively, ten cases $(25 \%)$ again developed an increase in the central macular thickness (CMT). Mean CMT decreased significantly from a baseline of $504.85 \pm 109.05 \mu \mathrm{m}$ to $341.10 \pm 75.35 \mu \mathrm{m}$ at 3 months and $322.05 \pm 87.40 \mu \mathrm{m}$ at 6 months, as tabulated in Table (3) and illustrated in Fig (2).

Assessment of visual acuity during the 6 months follow up showed a significant improvement in VA postoperatively in all patients by more than 2 lines in snellen chart at 3 months except in 6 eyes where the improvement of VA was minimal . at the $6^{\text {th }}$ month, of total, 24 eyes showed a significant improvement in VA by more than 2 lines in snellen chart. Mean BCVA showed statistically significant improvement from a baseline of $0.08 \pm 0.05$ decimal to $0.3 \pm 0.13$ decimal at 3 months and $0.35 \pm 0.16$ decimal at 6 months, as tabulated in Table (4) and illustrated in Fig (3).

HbA1c had significant negative correlation with the $6^{\text {th }}$ month BCVA, but significant positive correlation with the $6^{\text {th }}$ month CMT, as tabulated in Table (5).

An Example of a patient's serial OCT macula \& FFA is shown in Fig $(4,7)$.

Table (1) Patients' characteristics.

\begin{tabular}{ll}
\hline Variable & Values \\
\hline Gender: & $18(56.25 \%)$ \\
Male & $14(43.75 \%)$ \\
Female & \\
Eye laterality (No.) & \\
OD & $19(47.5 \%)$ \\
OS & $21(52.5 \%)$ \\
Age in years (mean \pm SD, range) & $57 \pm 7,42-71$ \\
Fasting blood sugar (mg/dl) (mean \pm SD, & $286.0 \pm 84.68,145.0-500.0$ \\
range) (mean \pm SD, range) & \\
HbA1c & $7.65 \pm 0.98,6.0-9.80$ \\
\hline
\end{tabular}


Table (2) IOP (mmHg) of the patients at all-time measures.

\begin{tabular}{llllllll}
\hline Variable & Preop & $\mathbf{1}^{\text {st }}$ month & $\mathbf{3}^{\text {rd }} \mathbf{~ m}$ & $\mathbf{6}^{\text {th }}$ month & F test & P value & Post Hoc \\
\hline IOP $(\mathrm{mmHg})$ & $13.70 \pm$ & $16.40 \pm$ & $14.20 \pm$ & $14.30 \pm 1.87$ & 9.64 & 0.001 & P1 0.006 \\
& 1.82 & 4.53 & 1.93 & & & & P2 1.00 \\
& & & & & & & P3 1.00 \\
& & & & & & P4 0.017 \\
& & & & & & P5 0.011 \\
& & & & & P6 1.00 \\
\hline
\end{tabular}

Table (3) CMT $(\mu \mathrm{m})$ of the patients at all-time measures.

\begin{tabular}{ll}
\hline Variable & CMT $(\boldsymbol{\mu m})$ \\
\hline Preop. & $504.85 \pm 109.05$ \\
3rd month & $341.10 \pm 75.35$ \\
6th month & $322.05 \pm 87.40$ \\
Test of significance & 103.93 \\
P value & $<0.001$ \\
Post Hoc & P1 $<0.001$ \\
& P2 $<0.001$ \\
& P3 0.271 \\
\hline
\end{tabular}

Table (4) VA (decimal) of the patients at all-time measures.

\begin{tabular}{ll}
\hline Variable & $\begin{array}{l}\text { VA } \\
\text { (decimal) }\end{array}$ \\
\hline Preop. & $0.08 \pm 0.05$ \\
3rd month & $0.3 \pm 0.13$ \\
6th month & $0.35 \pm 0.16$ \\
Test of significance & 87.42 \\
P value & $<0.001$ \\
Post Hoc & $\mathrm{P} 1<0.001$ \\
& $\mathrm{P} 2<0.001$ \\
& $\mathrm{P} 30.038$ \\
\hline
\end{tabular}

Table (5) Correlation between HbA1c and 6th month VA and CMT.

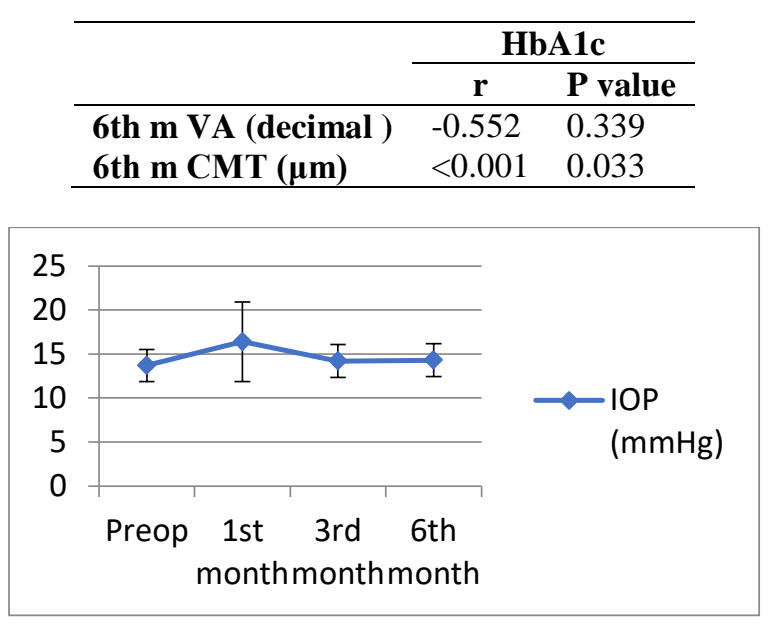

Fig (1) IOP (mmHg) at all time measures.

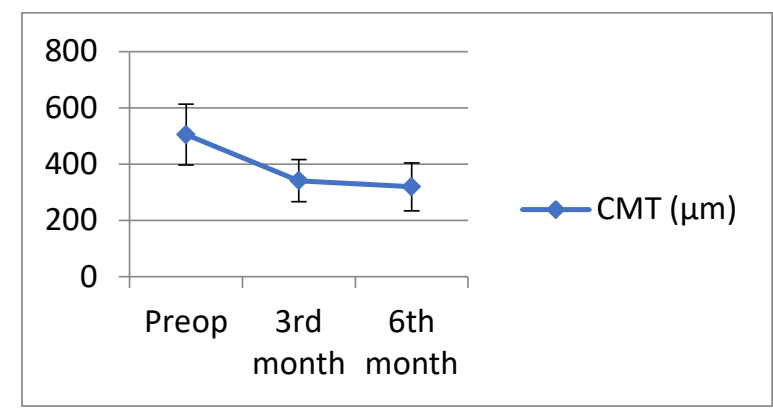

Fig (2) Mean CMT $(\mu \mathrm{m})$ of the patients at all-time measures.

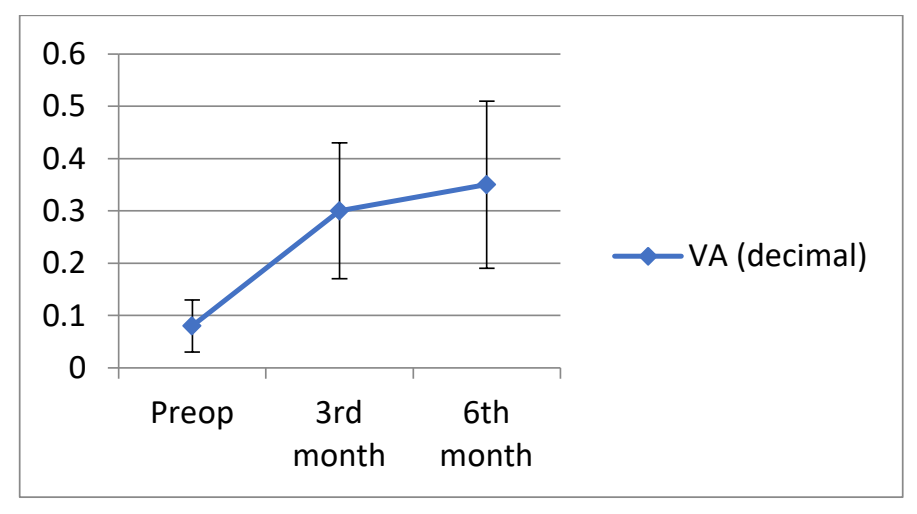

Fig (3) Mean BCVA (decimal) of the patients at all-time measures 


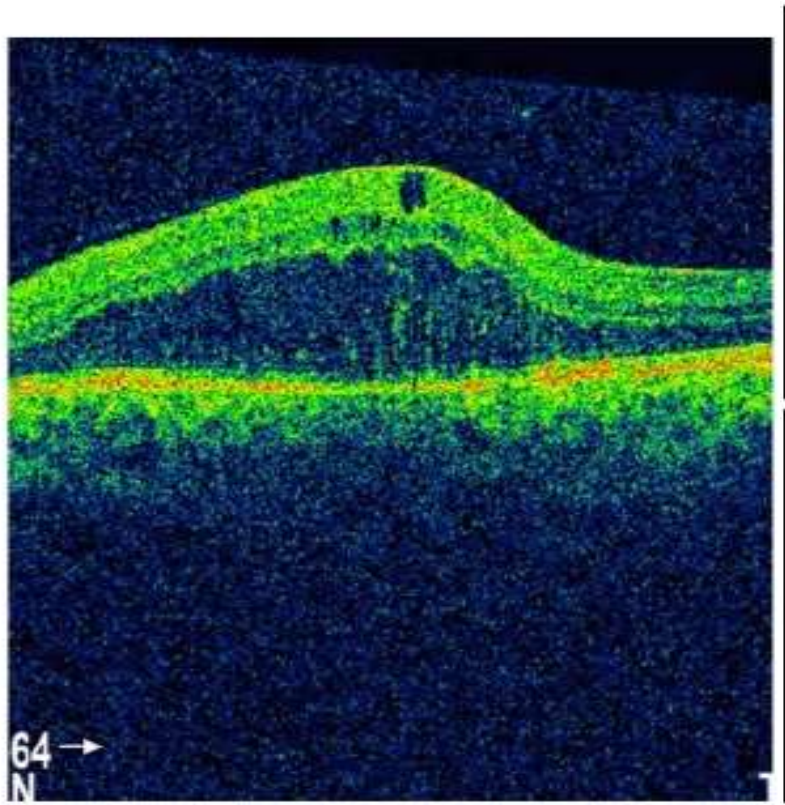

Fig (4) Preoperative OCT macula of a patient in the study with CME 1 week preoperatively with increased macular thickness (607 um).

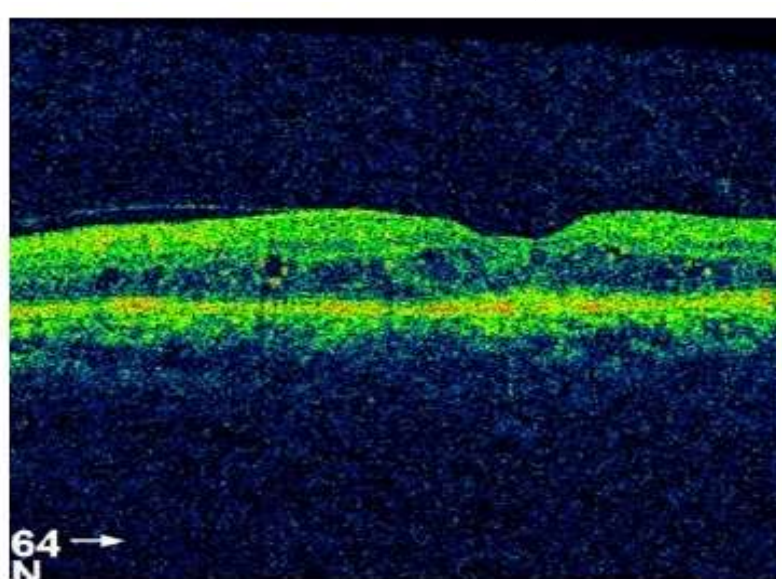

Fig (6) OCT macula of the same patient three months postoperatively shows dramatic resolution of the macular edema to (304 um).

\section{Discussion}

In patients with CSMO distinguished preoperatively, treatment with macular laser photocoagulation is prescribed in any event 12 weeks preceding medical procedure. Be that as it may, this might be troublesome in patients with huge waterfall. Diabetic CSMO stubborn to customary laser treatment strategies is likewise not an extraordinary issue. A great part of the current information in the writing propose that diabetic patients with CSMO at the hour of waterfall medical procedure have less fortunate visual results [9].

In [10] study, all patients with CSMO at the hour of medical procedure had diligent macular edema at the 1-year catch up with more unfortunate visual result (10). Likewise, the advancement of a part of
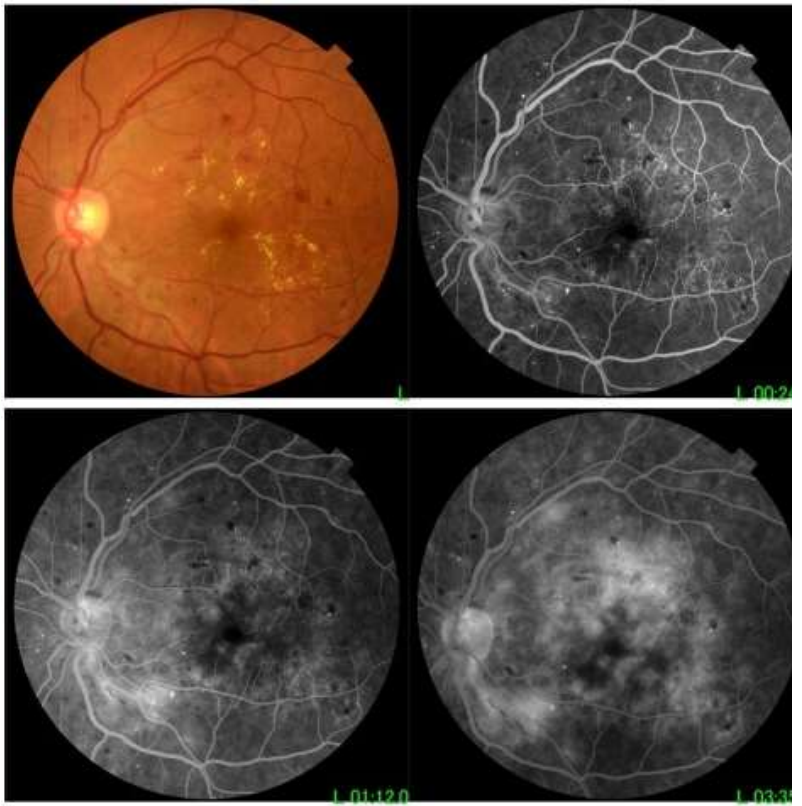

Fig (5) Preoperative FFA of the same patient 1 week preoperatively.

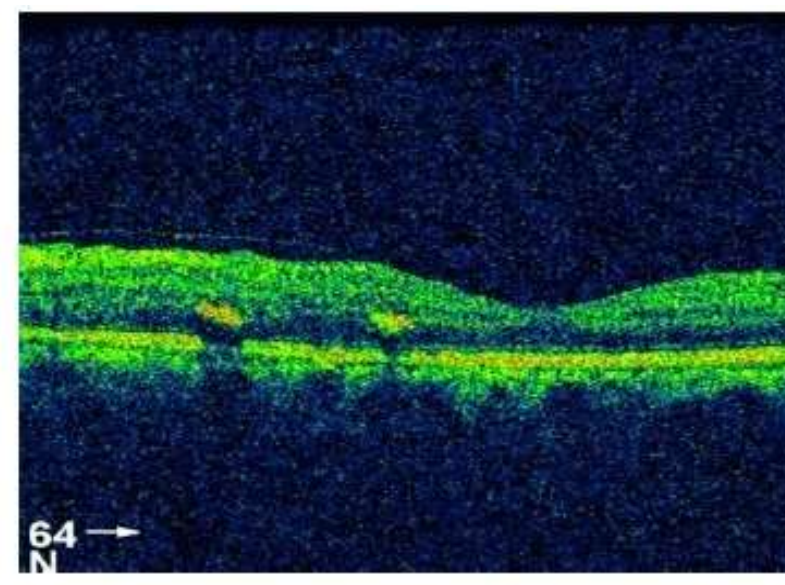

Fig (7) OCT macula of the same patient 6 months post operatively still showing resolution of the macular edema \& within normal thickness

(225um).

pseudophakic cystoid macular oedema (Irvine-Gass condition) is a potential jumbling factor and might be progressively basic in diabetic patients experiencing waterfall extraction when contrasted with the rate in non-diabetics [11].

Intravitreal pharmacotherapies have generally supplanted photocoagulation for patients with focus including DME. Corticosteroids are desirable over enemy of VEGF specialists in chose cases, patients who wish to diminish the quantity of required infusions and in pregnant patients who require treatment of the DME before conveyance [12].

[13] analyzed the viability of the IVTA versus intravitreal bevacizumab(IVB) in the treatment of DME. Consequences of IVTA bunch indicated 
noteworthy abatement in CMT when contrasted and IVB [13].

[14] examined forty eyes of 20 patients with twosided diffuse DME. For every patient, $4 \mathrm{mg} / 0.1 \mathrm{ml}$ IVTA was infused to one eye and $2.5 \mathrm{mg} / 0.1 \mathrm{ml} \mathrm{IVB}$ was infused to the next eye. The impacts of infusion for diabetic macular edema (DME) were assessed utilizing BCVA, CMT by OCT and IOP by applanation tonometer. Follow-up was done 1, 4, 8, 12 and 24 weeks after infusion. The outcomes indicated visit macular edema repeats in the eyes treated with bevacizumab contrasted and the ones treated with triamcinolone acetonide. Triamcinolone acetonide was found to give progressively proficient and long-standing impact as far as lessening CMT contrasted and the bevacizumab [14].

The current investigation was directed on 40 eyes of 32 patients having clinically critical diabetic macular edema and youthful decrepit waterfall treated by joined phacoemulsification and intravitreal infusion of $4 \mathrm{mg}$ triamcinolone acetonide (IVTA) to look at practical and focal thickness changes of the macula for a half year.

Everyone's eyes remembered for the examination had simple phacoemulsification with unblemished back case, flawless zonules and nonattendance of vitreous herniation with intrabagal PCIOL implantation.

Intravitreal infusion of TA has been related with different potential entanglements, height of IOP is the most widely recognized. In our investigation, the mean preoperaitive IOP was $13.70 \pm 1.82(\mathrm{mmHg})$. One month after infusion, the mean IOP essentially raised to $16.40 \pm 4.53(\mathrm{mmHg})$, notwithstanding, at the third month, it was fundamentally diminished to $14.30 \pm 1.93(\mathrm{mmHg})$ to be non-critical from the preoperative level. The degree of IOP kept on being steady at $14.30 \pm 1.87(\mathrm{mmHg})$ till the sixth postoperative month. 6 patients $(15 \%)$ in our examination created raised IOP, from a gauge mean of $14+2 \mathrm{mmHg}$ to a mean of $27.33+2.52 \mathrm{mmHg}$ one month after infusion, which was controlled with topical antiglaucomatous drugs and just a single patient expected to proceed on antiglaucoma meds after the finish of the investigation.

The most genuine potential complexity of intravitreal TA infusion is the improvement of endophthalmitis [15]. Revealed a few instances of endophthalmitis after intravitreal TA infusion. It was recommended that diabetes because of its relationship with immunosuppression, might be a hazard factor for endophthalmitis after intravitreal TA infusions [15]. None of our patients in this investigation built up this genuine confusion postoperatively.

As to investigation in our examination, focal macular thickness (CMT) as estimated by OCT diminished from an underlying pretreatment mean of $504.85 \pm 109.05 \mu \mathrm{m}$ to a mean of $341.10 \pm 75.35 \mu \mathrm{m}$ following multi month , at that point a mean of 322.05 \pm 87.40 at the sixth month. the focal macular oedema of everyone's eyes showed critical decrease in CMT up to the sixth month postoperatively aside from in 12 eyes (30\%). 10 eyes of those 12 eyes demonstrated decrease of CMT at the third month yet CMT expanded again at the sixth month in spite of the fact that not arriving at preoperative level. 2 eyes in the examination demonstrated recalcitrant oedema with unimportant decrease of CMT all through the investigation. Repeat of macular oedema following IVTA infusion isn't exceptional. Altogether, 37.5\% of eyes were found to have repeat of macular oedema a half year after IVTA infusion for headstrong diabetic macular oedema in the examination by [16].

With respect to in best remedied visual sharpness (BCVA) in the current examination, Mean BCVA was $0.08 \pm 0.05$ at gauge and improved to a mean of $0.3 \pm 0.13$ after 3 months , at that point to a mean of $0.35 \pm 0.16$ at the sixth month after medical procedure. In our examination, 24 eyes $(60 \%)$ Of the 40 eyes, had critical improvement in BCVA by in excess of 2 snellen lines at a half year. 14 eyes had improvement in BCVA under 2 snellen lines at 6 months (10 eyes indicated BCVA disintegration at the sixth month when CMT expanded toward the finish of the investigation and 4 eyes with noteworthy CMT decrease yet negligible improvement in BCVA). 2 eyes with headstrong macular oedema demonstrated no improvement in BCVA all through the investigation.

[17] considered 19 eyes of 15 diabetic patients with waterfall and CSMO. Patients experienced phacoemulsification and intraocular focal point implantation with $4 \mathrm{mg}$ IVTA infusion at fruition of medical procedure. The impacts of infusion for diabetic macular edema (DME) were assessed utilizing BCVA, CMT by OCT and IOP by applanation tonometer. Patients were followed up on day 1 , at that point week after week for multi month, and from that point month to month until a half year postoperatively. Altogether, 17 eyes finished a half year of development and results demonstrated $58.8 \%$ improvement in BCVA of $\geqslant 2$ lines, with measurably critical improvement in mean Snellen BCVA of 2.4 lines at a half year. The mean CMT diminished from a gauge of $449 \mu \mathrm{m}$ to at least $321 \pm 148 \mu \mathrm{m}$ accomplished at 2 months, with measurably huge decrease at all postoperative time interims until a half year. Of 17 eyes, $4(23.5 \%)$ grew temporarily raised intraocular pressure that standardized by a half year in everything except one patient. No infusion or medical procedure related intricacies were encountered [17].

[18] Examined 41 eyes of 36 diabetic patients with waterfall and coinciding clinically noteworthy macular odema (CSMO). After PHACO and IVTA infusion eyes were separated into two gatherings: the laser and IVTA gathering (Group 1) and just IVTA gathering (Group 2). Results indicated that postoperative BCVA was essentially higher than the underlying BCVA during the subsequent period in the two gatherings. The BCVA a half year after 
medical procedure was fundamentally higher in bunch 1 than in bunch 2 . There was no factually huge contrast between the two gatherings in mean CMT preoperatively and second week, second month and third month after medical procedure. The mean CMT a half year after medical procedure was measurably altogether lower in bunch 1 than in bunch 2 [18].

The genuine degree of the commitment of more clear media after waterfall extraction in improving visual keenness of those patients with diabetic CSMO is hard to find out, as we didn't have a benchmark group in our examination contrasting diabetic patients and CSMO who experienced phacoemulsification without IVTA. By the by, anatomical improvement in macular oedema has recently been demonstrated to be exceptionally related with useful improvement in visual sharpness in different examinations utilizing IVTA alone $[13,14]$.

\section{Conclusion}

Phacoemulsification with infusion of intravitreal TA might be performed securely in DME patients without danger of huge difficulties, for example, steady IOP rise and endophthalmitis. Also, $70 \%$ of eyes had measurably huge CMT decrease with $60 \%$ critical improvement in BCVA postoperatively, which was kept up a half year of development. Notwithstanding, further multicenter concentrates on a bigger example size are expected to evaluate the drawn out adequacy and the requirement for retreatment or utilizing IVTA as adjuvant to different specialists.

\section{Conflict of interest}

The authors have no conflict of interest to declare.

\section{References}

[1] J.Ding, T.Y.Wong, Current Epidemiology of Diabetic Retinopathy and Diabetic Macular Edema. Current Diabetes Reports. August, Vol.12, PP.346-354. 2012

[2] B.Wirostko , T.Y.Wong , R.Simó ,Vascular endothelial growth factor and diabetic complications. Progress in Retinal and Eye Research ,Vol.27, PP.608-6212008.

[3] T .Murakami , N .Yoshimura, Structural Changes in Individual Retinal Layers in Diabetic Macular Edema. Journal of Diabetes Research; Article ,Vol.27, PP.608-621 2013.

[4] M .Sahin, A.K.Cingü, N.Gözüm, Evaluation of cystoid macular edema using optical coherence tomography and fundus autofluorescence after uncomplicated phacoemulsification surgery. Journal of Ophthalmology,Volume Article ID ,Vol.27, PP.608-621 2013

[5] J.Liu, R.E.Jones, J.Zhao, Influence of Uncomplicated Phacoemulsification on Central
Macular Thickness in Diabetic Patients: A MetaAnalysis. PLoS ONE; Vol.10, PP.12-343. 2015

[6] M.W Stewart,Corticosteroid Use for Diabetic Macular Edema: Old Fad or New Trend? . Current Diabetes Reports, August, Vol.12, PP.364-375 2012

[7] Campos António, Beselga.Diana, Mendes.Sílvia, Journal of Ocular Pharmacology and Therapeutics. November, Vol.30, PP. 717-728. 2014

[8] M.S.Habib , P.S.Cannon , D.H.Steel ,The combination of intravitreal triamcinolone and phacoemulsification surgery in patients with diabeticfoveal oedema. BMC Ophthalmology ,Vol.10, PP.12-303,2005

[9] Royal.College of Ophthalmologists, The Royal College of Ophthalmologists Published Guidelines: Cataract Surgery Guidelines ; Vol.10, PP.11-3332001.

[10] J.G.Dowler, Kulwant SS, Hykin.PG and Hamilton.PA, The natural history of macular edema after cataract surgery in diabetes. Ophthalmology; 106: 663-668. 1999

[11]S.Ray , D.J, D’Amico, Pseudophakic cystoid macular edema. Semin Ophthalmol,Vol.17: , PP.167-1802002.

[12] S.G. Schwartz, I.U.Scott , M.W.Stewart,Update on corticosteroids for diabetic macular edema. Clinical Ophthalmology (Auckland, NZ),Vol.10,pp1723-1730. 2016

[13] X.L.Zhang, J.Chen , R.J.Zhang , Intravitreal triamcinolone versus intervitreal bevacizumab for diabetic macular edema: a meta-analysis. Int J Ophthalmol,Vol.6, pp546-552, 2013

[14] S.Aksoy, G.Yilmaz , I .Akkoyun, Comparison of intravitreal bevacizumab and triamcinolone acetonide therapies for diffuse diabetic macular edema. International Journal of Ophthalmology,Vol. 8, PP.550-555,2015

[15]D.M.Moshfeghi, P.K.Kaiser, I.U.Scott, Acute endophthalmitis following intravitreal triamcinolone acetonide injection. Am J Ophthalmol,Vol. 136, PP. 791-796, 2003

[16] A.Martidis, J.Duker, P.Greenberg, Intravitreal triamcinolone for refractory diabetic macular edema: Ophthalmology,Vol.109, pp.920-927, 2002

[17]D.S.Lam, C.K.Chan ， S.Mohamed, Phacoemulsification with intravitreal triamcinolone in patients with cataract and coexisting diabetic macular oedema: a 6-month prospective pilot study. Eye (Lond) 2005; 19:885-890.

[18] O.R.Ozgur, Y. Ozkurt, Z.Kulekci , The combination of phacoemulsification surgery and intravitreal triamcinolone injection in patients with cataract and diabetic macular edema. Saudi Journal of Ophthalmology Volume 30, Issue 1, January-March, Pages,Vol.11, PP.33-38,201 